\title{
Observation of light-by-light scattering and search for axion-like particles with the CMS experiment
}

\author{
Rebeka Lilla Tamási-Böttger ${ }^{a, b, *}$ \\ ${ }^{a}$ on behalf oft he CMS Collaboration \\ ${ }^{b}$ MTA-ELTE Lendület CMS Particle and Nuclear Physics Group, Eötvös Loránd University, \\ Pázmány Péter sétány 1A, Budapest, H-1117, Hungary \\ E-mail: bottger.rebeka@gmail.com
}

Evidence for the light-by-light scattering process, $\gamma \gamma \rightarrow \gamma \gamma$, in ultraperipheral $\mathrm{PbPb}$ collisions at a centre-of-mass energy per nucleon pair of $5.02 \mathrm{TeV}$ was reported by the CMS Collaboration at the LHC based on a data sample corresponding to an integrated luminosity of $390 \mu \mathrm{b}^{-1}$. Light-by-light scattering processes were selected from the events in which only two photons are exclusively produced, each with transverse energy $E_{T}^{\gamma}>2$ $\mathrm{GeV}$, pseudorapidity $\left|\eta^{\gamma}\right|<2.4$, diphoton invariant mass $m^{\gamma \gamma}>5 \mathrm{GeV}$, diphoton transverse momentum $p_{T}^{\gamma \gamma}<1 \mathrm{GeV}$, and diphoton acoplanarity below $\mathrm{A}<0.01 .14$ events were observed after all selection criteria, compared to expectations of $9.0 \pm 0.9$ (theo) events for the signal and $4.0 \pm 1.2$ (stat) for the background processes. The excess observed in data relative to the background-only expectation corresponds to a significance of 3.7 standard deviations and has properties consistent with those expected for the light-by-light scattering signal. The measured fiducial light-by-light scattering cross section, $\sigma_{\text {fid }}(\gamma \gamma \rightarrow \gamma \gamma)=120 \pm 46$ (stat) \pm 28 (syst) \pm 12 (theo) nb, is consistent with the Standard Model prediction. The $m^{\gamma \gamma}$ distribution was used to set constraints on the production of pseudoscalar axion-like particles via the $\gamma \gamma \rightarrow \mathrm{a} \rightarrow \gamma \gamma$ process in the mass range $m_{a}=5-90 \mathrm{GeV}$.

40th International Conference on High Energy physics - ICHEP2020

July 28 - August 6, 2020

Prague, Czech Republic (virtual meeting)

\footnotetext{
${ }^{0}$ The author wishes to thank for their support the Hungarian Academy of Sciences "Lendület" (Momentum) Program (LP 2015-7/2015) and the National Research, Development and Innovation Office of Hungary (K124845 and K128713).

* Speaker
} 


\section{Introduction}

The axion is a hypothetical particle which was introduced to solve the strong CP problem of the Standard Model and its mass depends on the coupling strengths to the Standard Model particles. An extension of the idea leads to axion-like particles (ALPs) whose mass and coupling strengths are free parameters. ALPs are not necessarily linked to the strong CP problem and arise in the spontaneous breaking of a global symmetry. The mass and couplings of ALPs can span many orders of magnitude. They can either be a component of dark matter or a mediator to a dark sector.

\section{ALP at the LHC}

Axion-like particles can be observed in ultraperipheral interactions of ions [1], where the impact parameter is larger than twice the radius of the nucleus. Accelerating ions at high energy produces strong electromagnetic fields which can be considered as $\gamma$ beams. Since each photon flux scales with the square of the ion charge $\boldsymbol{Z}^{2}$, the $\gamma \gamma$ final state scattering cross section in $\mathrm{PbPb}$ collisions are enhanced by a factor of $\mathbf{Z}^{\mathbf{4}} \approx \mathbf{5} \cdot \mathbf{1 0}^{\mathbf{7}}$ compared to the proton-proton and electron-positron interactions at the same

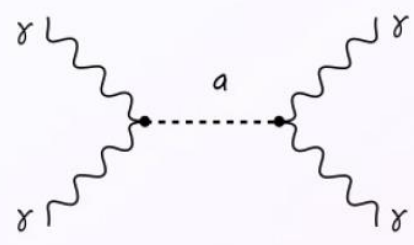

Figure 1. Feynman diagram of axion production via $\gamma \gamma \rightarrow a \rightarrow \gamma \gamma$. center-of-mass energy.

In the search for axion production $(\gamma \gamma \rightarrow \mathrm{a} \rightarrow \gamma \gamma)$, the dominant background comes from three processes: light-by-light (LbL) scattering $(\gamma \gamma \rightarrow \gamma \gamma)$; QED production of an exclusive electron-positron pair $\left(\gamma \gamma \rightarrow \mathrm{e}^{+} \mathrm{e}^{-}\right)$, where the electron and positron are misidentified as photons; and finally the gluon-induced central exclusive production (CEP) of a pair of photons ( $g g \rightarrow \gamma \gamma$ ).
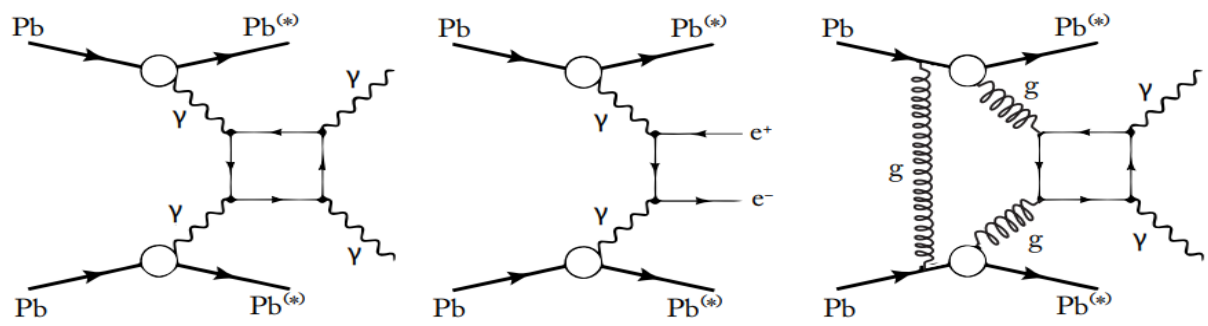

Figure 2. Feynman diagrams of background processes in the search for axion production.

\section{Investigation of $\mathrm{LbL}$ scattering}

Evidence for the LbL scattering process in ultraperipheral $\mathrm{PbPb}$ collisions at a centre-of-mass energy per nucleon pair of $5.02 \mathrm{TeV}$ was reported by the CMS Collaboration in Ref. [2]. The analysis was conducted using a data sample corresponding to an integrated luminosity of 390 $\mu \mathrm{b}^{-1}$. The two dominant background processes which contribute to the reconstructed diphoton final state in addition to LbL scattering were the QED dielectron and CEP diphoton productions.

Events with two exclusively produced photons each with transverse energy of $\boldsymbol{E}_{\boldsymbol{T}}^{\boldsymbol{\gamma}}>2$ $\mathrm{GeV}$, pseudorapidity of $\left|\boldsymbol{\eta}^{\boldsymbol{\gamma}}\right|<2.4$ were selected. The diphoton invariant mass had to satisfy $\boldsymbol{m}^{\gamma \boldsymbol{\gamma}}>5 \mathrm{GeV}$, and the diphoton transverse momentum $\boldsymbol{p}_{\boldsymbol{T}}^{\gamma \boldsymbol{\gamma}}<1 \mathrm{GeV}$. The diphoton CEP and the light-by-light scattering processes have different kinematic distributions. In the signal process, the diphotons are produced almost at rest in the transverse plane, thus the final-state photons are 
emitted back-to-back, while CEP photon pairs are produced with larger momentum. The $\mathrm{A}<0.01$ requirement on the diphoton acoplanarity significantly reduces the CEP background. Its rate was normalised to match the data in the $\mathrm{A}>0.02$ region, where the contribution of the LbL signal process is negligible.

The QED dielectron production was estimated from Starlight simulation [3]. Fig. 3 shows the comparison of the Monte Carlo simulation to the data. A good agreement can be seen in the region $\mathrm{A}<0.01$, while at higher acoplanarities the measured data contains other processes.

\section{LbL distributions and ALP exclusion limit}

After all selection criteria were applied, 14 events

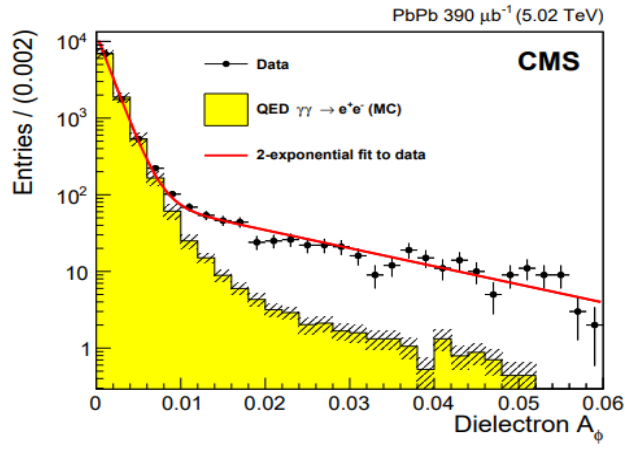

Figure 3. Distribution of dielectron acoplanarity for theoretical and measured data. were observed, compared to an expectation of $9.0 \pm$ 0.9 (theo) for the signal and $4.0 \pm 1.2$ (stat) for the background processes, as illustrated on Fig. 4. The measured ratio of the exclusive diphoton and dielectron yields is $R=(25.0 \pm$ 9.6 (stat) \pm 5.8 (syst) $) \times 10^{-6}$. The fiducial cross section is measured to be $\sigma_{\text {fid }}(\gamma \gamma \rightarrow$ $\gamma \gamma)=120 \pm 46$ (stat) \pm 28 (syst) \pm 12 (theo) $\mathrm{nb}$ and is consistent with the theoretical prediction obtained from Startlight simulation.
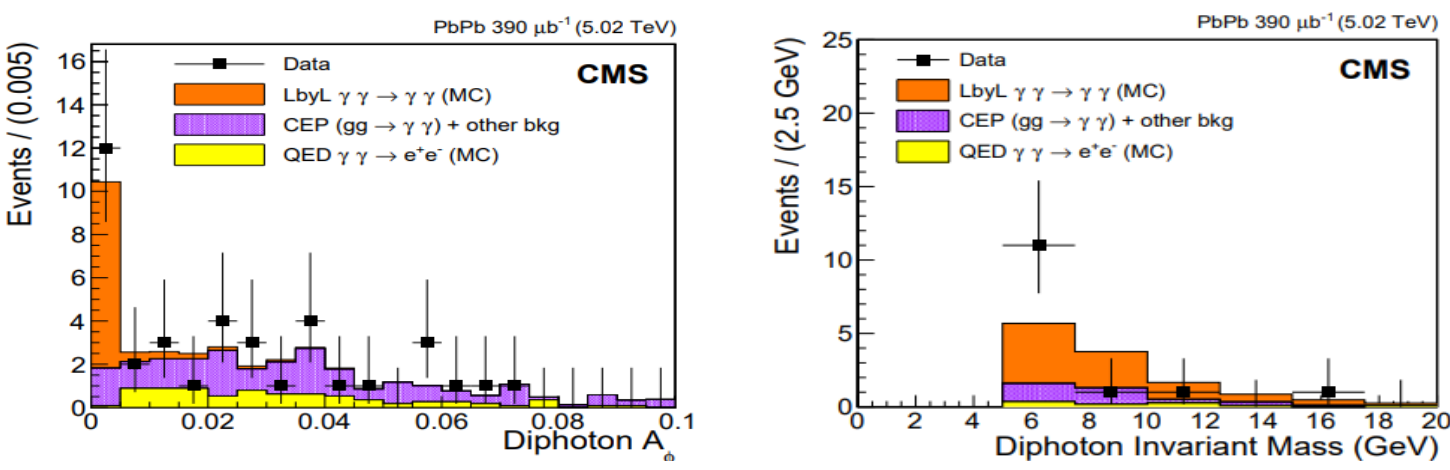

Figure 4. Distributions of the diphoton acoplanarity and for the region of $A<0.1$ the diphoton invariant mass.

Limits on the ALP production cross section were set in the 1500-20 nb range over masses between 5 and $90 \mathrm{GeV}$ as shown in Fig 5. The derived exclusion limits were the best at the time of publication and were interpreted in the mass coupling strength plane assuming coupling only to photons or via hypercharge also to $\mathrm{Z}$ bosons.

\section{References}

[1] S. Knapen, T. Lin, H.K. Lou, T. Melia, Phys. Rev. Lett. 118 (2017) 171801

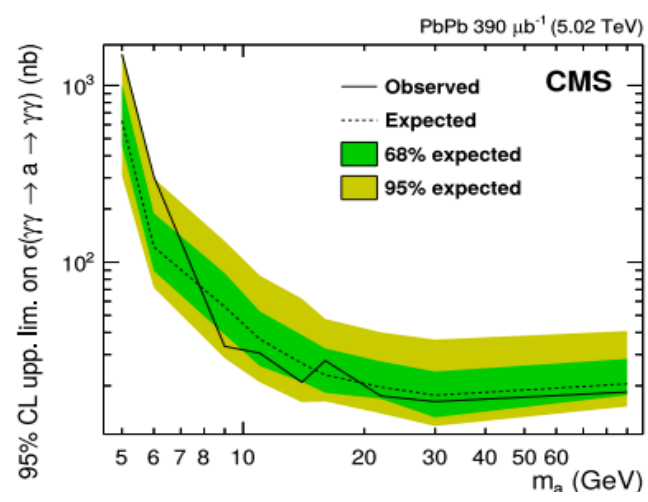

Figure 5. Limits on the ALP production cross section.

[2] CMS Collaboration, Phys. Lett. B 797 (2019) 134826

[3] S. R. Klein et al., Comput. Phys. Commun. 212 (2017) 258 